Radio Science, Volume 8, Number 11, pages 1035-1048, November 1973

\title{
Active experiments, magnetospheric modification, and a naturally occurring analogue
}

\author{
Margaret G. Kivelson and C. T. Russell \\ Institute of Geophysics and Planetary Physics, University of California, \\ Los Angeles, California 90024
}

(Received August 6, 1973.)

\begin{abstract}
Active experiments in the magnetosphere include some intended to be primarily diagnostic (barium release, electron guns) and others designed to perturb the system to generate instabilities (VLF or ULF wave injection from ground stations, ionospheric heating). Recently, a scheme has been proposed which would modify the magnetosphere by injecting plasma near the equator beyond the plasmapause and initiating wave-particle instabilities. The expected effects have been examined theoretically. Injection of plasma into this region is also a naturally occurring phenomenon produced by the cross-tail electric fields which are associated with geomagnetic activity. For further investigation of magnetospheric instabilities, the advantages of examining artificially injected plasma (control of time and location of injection and of the volume of plasma injected) contrast with the advantages of studying natural enhancements (no extra payload, frequent occurrence). Thus, the two types of experiments are complementary. In preliminary studies of natural plasma enhancements both ULF and ELF emissions have been observed. The ELF noise is consistent with generation by the electron cyclotron instability.
\end{abstract}

\section{INTRODUCTION}

During the 1960's the properties of the magnetosphere were investigated primarily with passive techniques, both ground-based and on satellites. In recent years, the methods of observation have been broadened to include active experiments in which the magnetosphere is locally modified and its response to a well-defined perturbation is examined. In this paper, we briefly describe a number of active experiments which have been performed or suggested, emphasizing the different ways in which they contribute to an understanding of the magnetosphere. We then concentrate on the proposed light ion seeding experiments, reviewing the anticipated effects of plasma injection in the magnetosphere beyond the plasmapause. Noting that plasma injection beyond the plasmapause can occur naturally, producing substantially similar phenomena, we report on preliminary observations in such naturally occurring plasma enhancements.

\section{BRIEF HISTORY OF ACTIVE EXPERIMENTS}

Active experiments which have been performed in the magnetosphere and the ionosphere or are

Copyright (C) 1973 by the American Geophysical Union. being considered are summarized in Table 1 . These experiments include the release of heavy ion plasma clouds and the injection of accelerated charged particles, as well as the injection of electromagnetic wave energy at frequencies ranging from ULF $(0.001$ to $10 \mathrm{~Hz}$ ) to $\mathrm{HF}$ ( 3 to $30 \mathrm{MHz}$ ). The charged particle experiments have been used to measure ambient electric fields and to verify conjugacy; beams of charged particles have also initiated instabilities, thus providing information on dynamical processes. The initiation of instabilities can be considered the principal purpose of most electromagnetic wave injection experiments, as well as of proposed light ion plasma seeding experiments.

2.1. Barium clouds. Plasma clouds of heavy ions, usually barium, have been released both in the ionosphere and deep in the magnetosphere [Foppl et al., 1965; Haerendel et al., 1967; Haerendel and Lüst, 1970; Brence et al., 1972]. At low altitudes, the drift motion has been used to infer electric fields [Haerendel, 1971a; Schutz et al., 1971] and to relate the observed electric fields to the dynamics of substorms [Haerendel, 1971a]. The development of striations in the barium clouds [Haerendel, 1971b] has demonstrated the importance of nonlinear processes. 
TABLE 1. Active experiments in the magnetosphere and the ionosphere.

\begin{tabular}{ccc}
\hline & \multicolumn{1}{c}{ Principal application } \\
\cline { 2 - 3 } & $\begin{array}{c}\text { Measurement of } \\
\text { preexisting fields; } \\
\text { verification of } \\
\text { conjugacy }\end{array}$ & $\begin{array}{c}\text { Perturbation } \\
\text { of system }\end{array}$ \\
\hline $\begin{array}{c}\text { Heavy ion plasma clouds } \\
\text { (Barium, Cesium) }\end{array}$ & $\mathrm{X}$ & \\
$\begin{array}{c}\text { Injection of accelerated } \\
\text { particles (shaped Ba } \\
\text { charges, accelerated } \\
\text { electrons) }\end{array}$ & $\mathrm{X}$ & $\mathrm{X}$ \\
$\begin{array}{l}\text { Injection of ULF, VLF, } \\
\text { MF and HF waves }\end{array}$ & & \\
$\begin{array}{l}\text { Light ion plasma clouds in } \\
\text { the magnetosphere }\end{array}$ & & $\mathrm{X}$ \\
\hline
\end{tabular}

One of the most ambitious injections of a barium cloud from a rocket was a release at $5 R_{E}$ altitude [Brence et al., 1972]. The motion of this cloud has been used to infer the local magnetospheric electric field $\left(0.7 \mathrm{mv} \mathrm{m}^{-1}\right)$ [Rieger et al., 1972] while a simultaneous balloon flight measured both electric fields and X-ray intensity at the foot of the field line in the ionosphere [Mozer, 1972]. These measurements provided an upper limit on the flux of energetic electrons precipitated by the cloud. If the particle acceleration occurred over regions greater than several thousand $\mathrm{km}$ in diameter at the barium cloud, less than $1 \%$ of the trapped flux of electrons greater than $30 \mathrm{kev}$ was precipitated. On the other hand, if the interaction region was equal to the optical dimension of the cloud, of the order of $100 \mathrm{~km}$, then the precipitated flux could have equalled the trapped flux without disagreeing with $\mathrm{X}$-ray observations. Field lines traced by the barium ions were used to test magnetospheric models [Barish and Roederer, 1972] with results which suggest that a ring current at the magnetic equator is present even for moderately quiet times. Finally, the development of striations in the magnetospheric barium cloud has been interpreted in terms of a flute instability [Haerendel et al., 1972].

The most distant barium release was made from the HEOS-1 spacecraft at $12.5 R_{E}$ on March 18 , 1969 [Haerendel and Lüst, 1970]. At such a large radial distance, where the plasma density is probably below $1 \mathrm{~cm}^{-3}$, the motion of the plasma cloud is largely independent of the motion of the ambient medium. In fact, Haerendel and Lüst estimate that
1 to $10 \mathrm{hr}$ would be required to destroy differential motion between the injected and natural plasmas. The barium cloud elongated along the direction of the magnetic field as measured by the HEOS-1 magnetometer and broke into striations. During the formation of one such striation, the development of a weak tail was observed. Using this tail to put limits on the velocity of the ambient plasma Haerendel et al. [1971] showed that the observed magnetic fluctuations in the polar cap were not consistent with either Hall or Pedersen currents but could be interpreted in terms of a field-aligned current system.

2.2. Particle acceleration experiments. Accelerated charged particles injected along the direction of the magnetic field have been used to trace field lines over thousands of $\mathrm{km}$ and to identify conjugate points with great accuracy. Zhulin et al. [1972] have reviewed the experiments of this type. Nearequatorial injection of accelerated electrons aimed upward along the magnetic field from Hawaii [Davis et al., 1973] produced an artificial aurora at the conjugate point. In analogous experiments with barium shaped-charge generated plasma jets, ion velocities of several $\mathrm{km} \mathrm{sec}^{-1}$ (to be contrasted with velocities of approximately $10 \mathrm{~m} \mathrm{sec}^{-1}$ in isotropic barium releases) made it possible to track ions optically for $7000 \mathrm{~km}[F u$ et al., 1973]. Different drift velocities were observed at conjugate points in the northern and southern hemisphere suggesting that magnetic field lines are not always equipotentials [Westcott et al., 1972, 1973].

At midlatitudes, accelerated electrons have been injected downwards along the magnetic field toward the near conjugate point to produce artificial auroras [Hess et al., 1971]. Pulsed electron beams reflected from mirror points in both hemispheres have been studied as a controlled population from which the behavior of naturally injected, trapped energetic electrons can be infered [Winckler et al., 1970]. In these experiments, whistler mode and other plasma waves were generated by the injected energetic electrons [Cartwright and Kellogg, 1970]. No evidence has been found for large instabilities affecting beam propagation [Hendrickson et al., 1970] though the beam intensity decreased after multiple reflections.

From auroral zone injection points $(L=6.5)$ barium shaped-charge generated plasma jets have traced field lines deep into the magnetosphere during quiet and disturbed times [Westcott et al., 1973]. Here, too, accelerated electrons have been injected 
both to study particle behavior [Hendrickson et al., 1973] and to probe the boundary of closed field lines [Hess et al., 1971].

2.3. Wave injection. Electromagnetic waves in different frequency ranges have been beamed into the ionosphere to investigate transmission, propagation, and instabilities. In transmission of VLF waves (10 to $25 \mathrm{kHz}$ ) between conjugate points, pulsations have been observed for signals of long duration [Helliwell and Bell, 1971]. Signals of less than 200 msec duration are not observed at the conjugate point [Dowden et al., 1973]. This finite wave train effect is not described by current theoretical models of whistler amplification. The observation that VLF signals transmitted from the conjugate hemisphere are abruptly cut off as a polar orbiter enters the plasmasphere has led to the suggestion that relatively simple narrow band VLF receivers on polar orbiting satellites could monitor the plasmapause and other density structures in the magnetosphere and the ionosphere [Katsufrakis et al., 1973]. Proposals to build large, ground-based current loops to produce ULF hydromagnetic waves of observable amplitude in the ionosphere have not yet been implemented [Fraser-Smith, 1973]. On the other hand, radar heating of the ionosphere has been used to produce parametric plasma instabilities whose growth and saturation have been analyzed theoretically [Goldman and DuBois, 1971].

2.4. Light ion injection. The active experiments which have been carried out in the past demonstrate that perturbation of the magnetosphere is an effective experimental approach. As a logical extension of previous investigations, light ion injection into the outer radiation belt could significantly supplement present experimental methods. The consequences of such a plasma-seeding experiment were first discussed by Brice [1970, 1971 a, 1971 b, 1972] and have been further explored by Cornwall [1972] and Cornwall and Schulz, [1973]. These authors have concluded that an increase in the cold plasma density at synchronous orbit from the normal value of $1 \mathrm{~cm}^{-3}$ to $3 \mathrm{~cm}^{-3}$ would permit both electron and proton cyclotron instabilities to develop in the outer radiation zone as schematically depicted in Figure 1. Their predictions are based on the observation that cyclotron resonance can occur only for particles whose energy exceeds a resonant energy proportional to $B^{2} / 8 \pi N$, the magnetic energy per particle. An enhancement of plasma density lowers the minimum resonant energy permitting lower

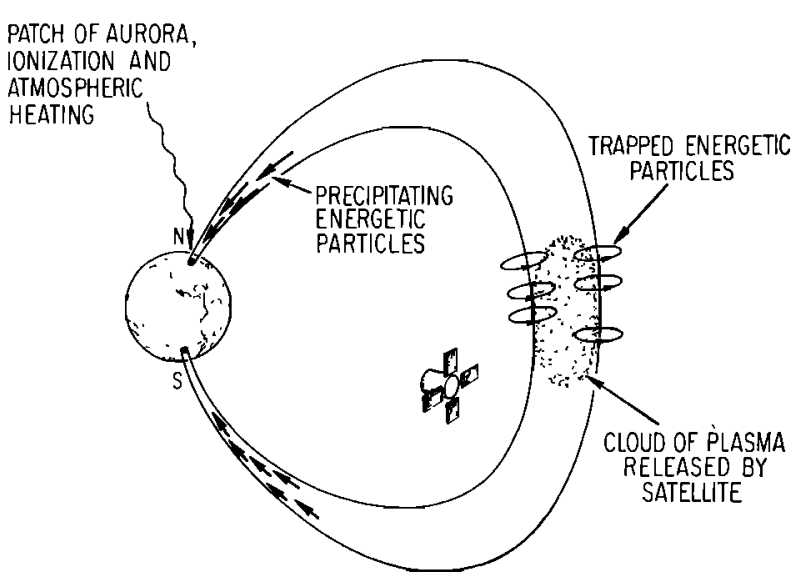

Fig. 1. Schematic view of a plasma injection experiment. (From Cornwall [1972].)

energy particles to become unstable. Cornwall and Schulz [1973] have estimated that a $50 \mathrm{~kg}$ payload will provide a sufficiently large lithium plasma cloud to produce observable effects, though the precipitated flux will increase with the dimension of the seeded volume. A light ion plasma is preferable to a heavy ion plasma both because the payload required is smaller and because a light ion is necessary for the proton cyclotron instability to develop. The effects anticipated include enhanced whistler-mode turbulence (VLF) and ion-cyclotron-mode turbulence (ULF), as well as the accompanying precipitation of energetic (greater than approximately $20 \mathrm{kev}$ ) electrons from the outer zone of trapped radiation and the precipitation of ring current protons (approximately $20 \mathrm{kev}$ ). Brice has suggested that the the plasma seeding may initiate a substorm [Brice, 1970] or speed up the recovery phase [Brice, 1971a]. Light ion plasma-seeding experiments would, as an additional dividend, extend the observations already made with barium plasma clouds. Field-line geometry could be investigated in such interesting regions as the nighttime cusp. Plasma diffusion rates along and across field lines could be measured and magnetospheric electric fields inferred.

\section{NATURAL PLASMA SEEDING}

Light ion injection into the outer radiation zone is also a naturally occurring phenomenon. In this case, the plasma consists mainly of hydrogen ions and the source of the plasma is detachment from the outer plasmasphere by time-varying electric fields. The proposed effects of plasma seeding should be independent of whether the source of the plasma 
is natural or artificial. Furthermore, wave and particle measurements already exist for such regions.

3.1. The injection mechanism. The detachment process has been described by Chappell, [1972] and we only briefly review it here. Figure 2, representing the equatorial plane, shows schematically equipotentials along which plasma flows in the presence of a dawn-dusk cross-tail electric field superimposed on the corotation field of the earth [Kavanagh et al., 1968]. Inside the boundary indicated with a heavy line the streamlines close on themselves and the plasma density can build up over many days. Outside of this boundary the plasma is swept away to the magnetopause. Thus, at the boundary which represents the plasmapause there is a sharp decrease in plasma density. Figures 3 and 4, similar schematic representations, indicate the effect of an increase in the cross-tail electric field [Chappell et al., 1971]. The boundary between closed and open streamlines, labelled $b$ in Figure 3, moves earthward to a position labelled $a$ in Figure 4 when the cross-tail field is increased. Plasma originally between boundaries $b$ and $a$ now is found on open streamlines and, in the afternoon sector, begins to flow toward the magnetopause, into regions in which the plasma density is very low. Near syn-

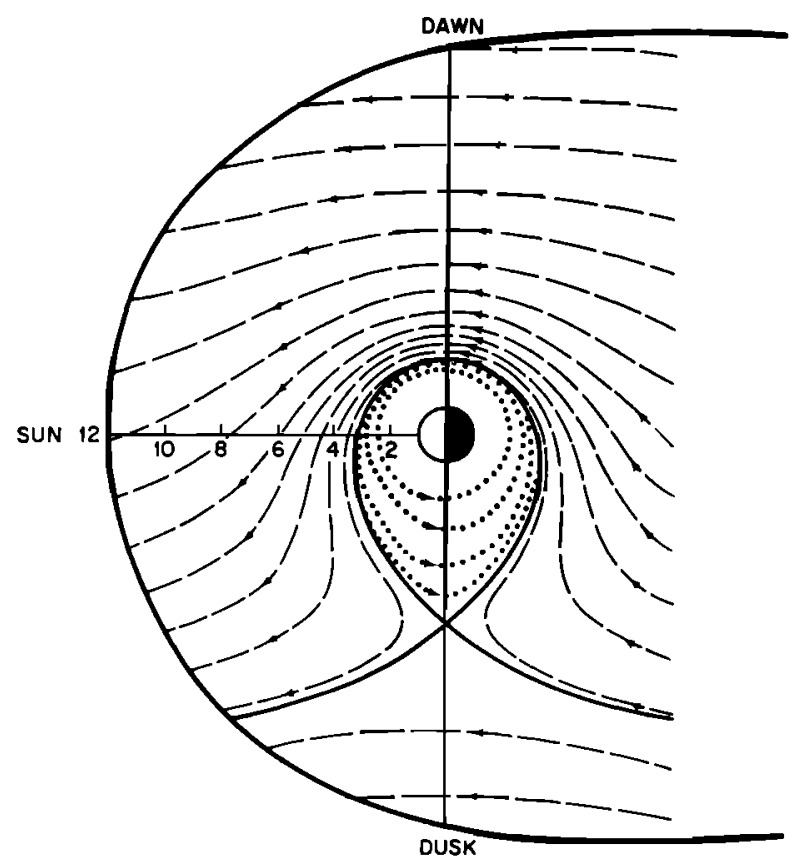

Fig. 2. Equipotentials or streamlines for thermal plasma in the presence of a cross-tail electric field. Outside the heavy contour, the flow is open to the magnetopause, the plasmasphere lies within. From Kavanagh et al. [1968].

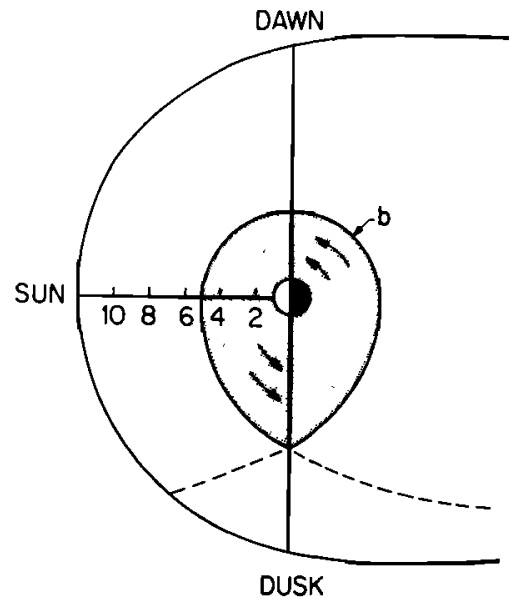

Fig. 3. Schematic representation of the formation of detached plasma: prestorm. The plasma within contour $b$ is on closed streamlines as in Figure 2.

chronous orbit the drift velocity at the equator of such a plasma enhancement is about $0.1 R_{E} \mathrm{~min}^{-1}$ in a field of $1 \mathrm{mv} \mathrm{m}^{-1}$. Plasma enhancements are observed to occur beyond the plasmapause, primarily in the afternoon, consistent with this model. However, it is important to recognize that if time variations in the cross-tail electric field occur on time scales of tens of minutes to hours, the same mechanism can produce isolated patches of enhanced plasma density beyond the plasmapause on the nightside as well.

Although the plasma of the plasmaspheric bulge also extends into the outer radiation zone near dusk, we emphasize the isolated plasma enhancements because they more nearly simulate the conditions for an artificial light ion seeding experiment. Energetic electrons can traverse the plasma bulge sufficiently often to produce a steady state. On the other hand, plasma-seeding experiments emphasize nonequilibrium conditions. The plasma enhancements are encountered mainly at larger $L$ than the average bulge position [Chappell, 1972] and the energetic electron flux in this region is not in equilibrium within the enhanced plasma. Furthermore, phenomena observed within the spatially limited enhanced plasma may be assumed to have a localized origin. For example, it is probable that wave turbulence observed in detached plasma enhancements has been produced within the enhancements, whereas waves observed in the plasma bulge may have propagated from the distant plasmasphere. 


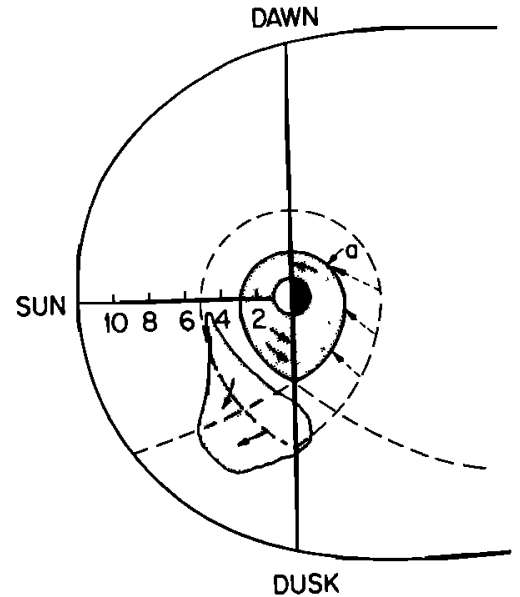

Fig. 4. The formation of detached plasma: during the storm. The crosstail field has been enhanced; closed flow lines are found within contour $a$. Plasma initially between $a$ and $b$ is now on open streamlines and moves into the afternoon quadrant. (From Chappell [1972].)

3.2. Natural or artificial seeding. Though the phenomena which should occur in regions of enhanced plasma density beyond the plasmapause are similar for similar conditions whether the injection mechanism be natural or artificial, both techniques have advantages and disadvantages. Artificially injected plasma can be placed at any desired location in the magnetosphere at a selected time, whereas natural injection occurs primarily in the afternoon quadrant and at random times. The artificial plasma clouds will have small spatial dimensions, and such injections will probably not be numerous, largely because of the payload required. Naturally occurring detached plasma enhancements will have varied spatial extension, though their sizes will be inaccurately known. However, they can be observed without any extra payload, and since they occur frequently, they can be observed on many occasions. In other words, the two types of experiments are complementary.

\section{OBSERVATIONS OF THE EFFECTS OF NATURAL SEEDING}

The most extensive study of the region of detached plasma in the outer magnetosphere has been made using the OGO-5 Lockheed Ion Mass Spectrometer [Chappell, 1972]. OGO-5 carried a comprehensive payload of field and particle experiments and we report here the measurements made by some of these instruments (the UCLA/JPL Search Coil Magnetometer, the TRW Electric Field Experiment, the UCLA Energetic Electron Spectrometer, and the UCLA Fluxgate Magnetometer) during traversals of some of the regions of detached plasma investigated by Chappell.

4.1. ELF magnetic fluctuations. The effect of naturally occurring plasma enhancements in the outer radiation zone on energetic electrons and electromagnetic waves has been examined in a preliminary study using data from a number of experiments on OGO-5 [Kivelson et al., 1972]. Figure 5 shows the ion density observed by the Lockheed Light Ion Mass Spectrometer on an outbound pass of OGO-5 on September 13, 1968. The measure-

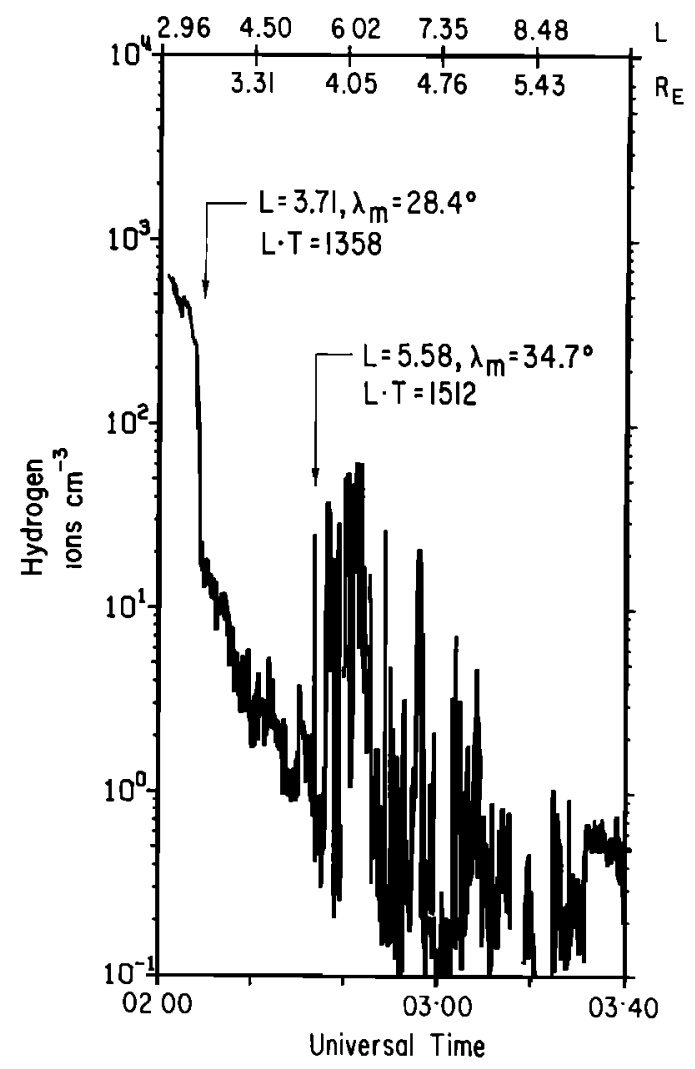

Fig. 5. Example of detached plasma density beyond the plasmapause; the diagram shows hydrogen ions measured by the Lockheed Light Ion Mass Spectrometer plotted with respect to universal time, September 13, 1968, OGO-5 outbound. The upper scale gives $L$-value and radial distance. $L$-values, magnetic latitude, $\lambda_{m}$, and local time are given for the plasmapause and the first enhancement. 
ments were made at magnetic latitudes near $35^{\circ}$ in the local time region between 1400 and $1600 \mathrm{hr}$. The plasmapause appears as a steep gradient in the number density at $L=3.7$, indicated by the first arrow. The enhanced plasma density was encountered almost half an hour later in the region from $L=5.6$ to $L=8$. Peak plasma densities fell off approximately as $L^{-4}$. The ragged appearance of the density enhancements may give evidence of instabilities analogous to those causing striations in barium clouds [Chappell, 1973].

The ELF spectra for this orbit measured by the

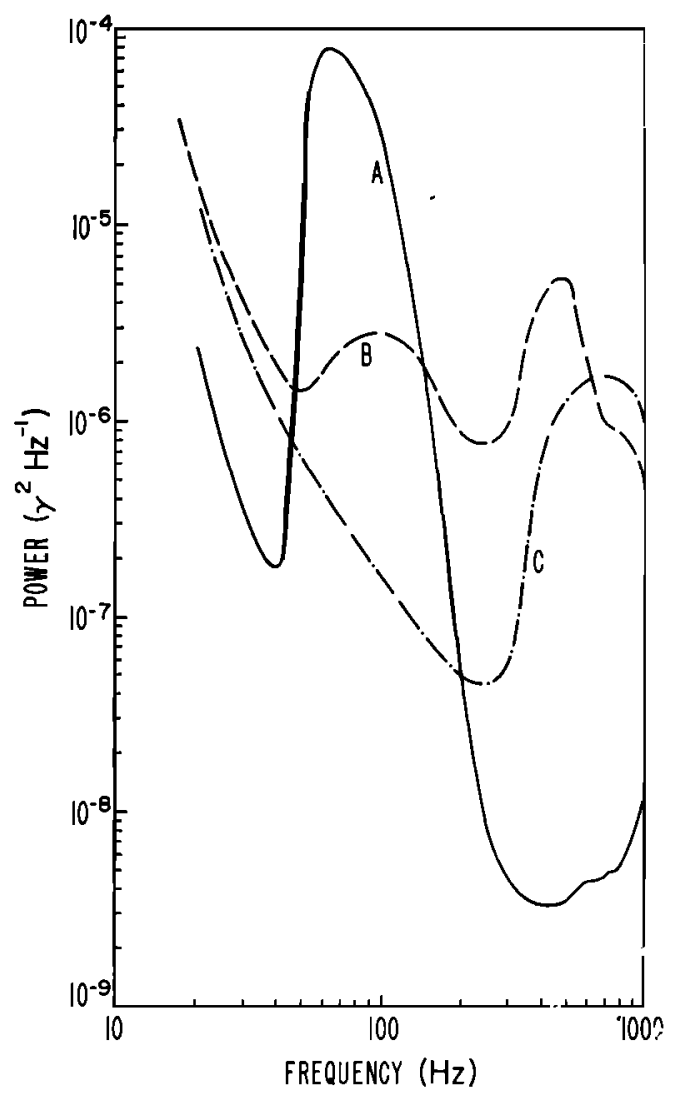

Fig. 6. Power spectra from 1-min averages of measurements of the UCLA/JPL Search Coil Magnetometer on OGO-5 for September 13, 1968. Curve $B$ (0200 UT) was measured within the plasmasphere where peaks are observed near 100 and $500 \mathrm{~Hz}$. Curve $C$ (0230 UT) was measured in the region of low plasma density beyond the plasmapause. This characteristic high latitude hiss has a peak just below $1000 \mathrm{~Hz}$ and little power near $100 \mathrm{~Hz}$. Curve $A$ (0236 UT), measured within a density enhancement, has peak power near $100 \mathrm{~Hz}$ and falls off at high frequency.

(From Chan et al. [1972].)
UCLA/JPL Search Coil Magnetometer have been described by Burton et al. [1972] and Chan et al. [1972] from which Figure 6 showing ELF power versus frequency is reproduced. The dashed trace, labelled $B$, is the spectrum measured at $0200 \mathrm{UT}$, within the plasmasphere. This plasmaspheric hiss terminated at the plasmapause, where a qualitatively different spectrum, here labelled $C$, with substantial power above $400 \mathrm{~Hz}$ was found. Burton et al. [1972] call this spectrum "high latitude hiss." Inside the detached plasma enhancements a spectrum labelled $A$ with peak power of order $10^{-4} \gamma^{2} \mathrm{~Hz}^{-1}$ near $100 \mathrm{~Hz}$, quite narrow banded in the range 50 to $150 \mathrm{~Hz}$, was observed.

Figure 7 shows ELF measurements for the same day on an expanded time scale for the interval during which plasma density enhancements were encountered. On the bottom panel the density of hydrogen ions is plotted versus universal time, with shading emphasizing those regions in which the ion density exceeds $4 \mathrm{~cm}^{-3}$. On the upper panel the spectrum channels from 10 to $999 \mathrm{~Hz}$ of a single axis of the UCLA/JPL Search Coil Magnetometer are plotted. Shading of the peaks in the $100 \mathrm{~Hz}$ channel emphasizes the high correlation between the presence of thermal plasma and of ELF signals at $100 \mathrm{~Hz}$. This close correlation supports the suggestion of Burton et al. [1972] that this low frequency band is generated by the cyclotron resonance of energetic electrons near the equator and is ducted through enhanced density regions to high latitudes. Measurements of the propagation direction show that the wave vector lies within less than $30^{\circ}$ of $B$. Note that at 0233 and 0256 UT, the plasma enhancements do not correlate with the $100-\mathrm{Hz}$ hiss. Note also that the high latitude hiss present in the 467 and $999 \mathrm{~Hz}$ channels is anticorrelated with the density enhancements, even in the narrow enhancement at 0233 UT.

One possible cause of the absence of $100-\mathrm{Hz}$ noise in these two enhancements is that the signals have leaked out because the enhancements were too narrow to trap the radiation. In particular, the narrowest enhancement at 0233 UT was traversed in $20 \mathrm{sec}$. A stationary enhancement of less than $84 \mathrm{~km}$ thickness would have been traversed in this time at the satellite velocity of $4.2 \mathrm{~km} \mathrm{sec}^{-1}$. Although the enhancement might be moving, its velocity should be less than that of the satellite. For example, the velocity corresponding to an electric field of $1 \mathrm{mv} \mathrm{m} \mathrm{m}^{-1}$ is $1.2 \mathrm{~km} \mathrm{sec}^{-1}$, in the observed 


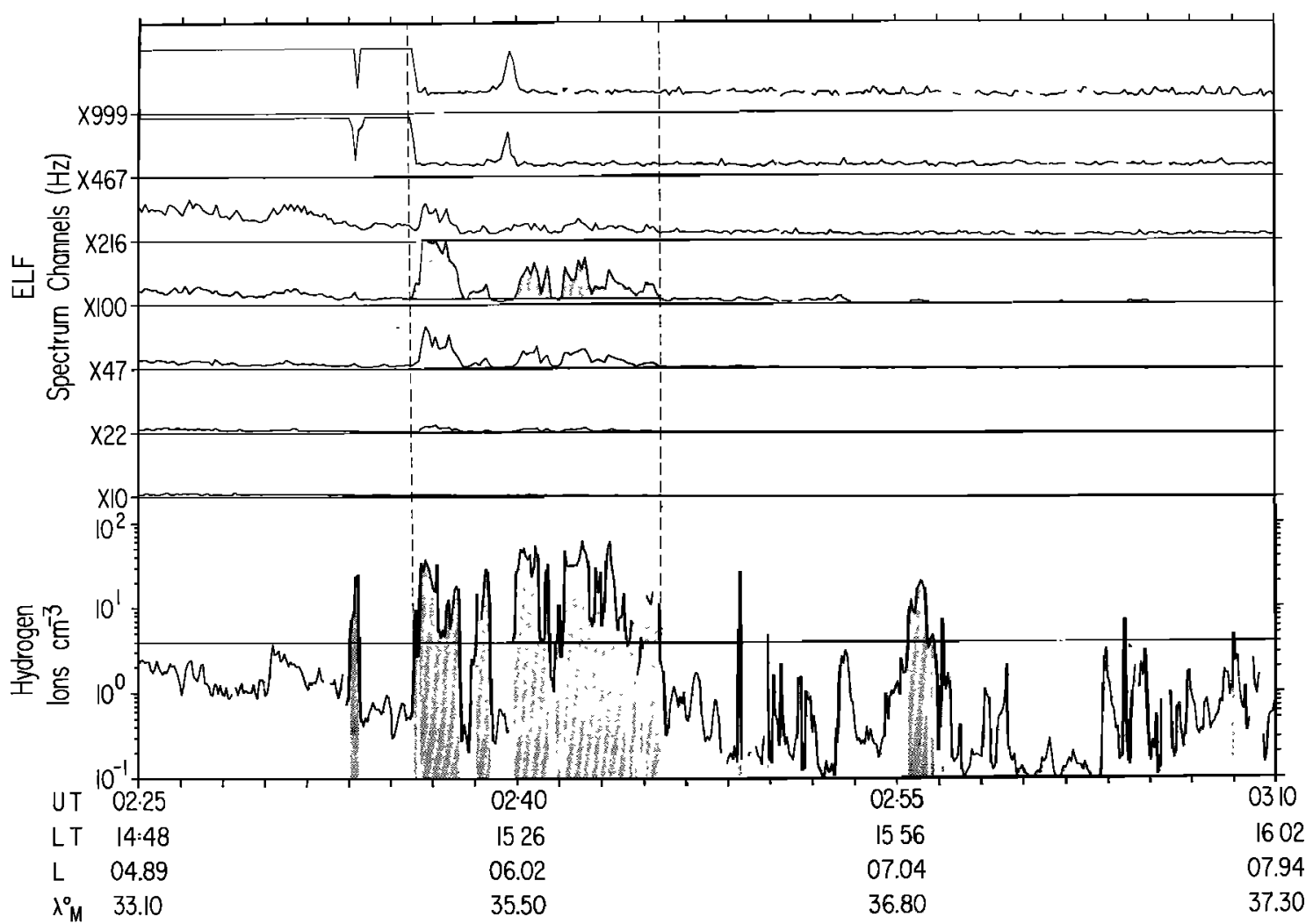

Fig. 7. Correlated ELF-hiss and plasma density enhancements observed by OGO-5 on September 13, 1968. The bottom panel displays the measurements of the Lockheed Light Ion Mass Spectrometer on an expanded time scale. The upper panels display the rms magnetic noise measured along the spacecraft $x$ axis by the UCLA/JPL Search Coil Magnetometer at seven frequencies from 10 to $999 \mathrm{~Hz}$. The intervals during which the plasma density exceeded $4 \mathrm{~cm}^{-3}$ have been shaded in the 100-Hz channel and in the ion density plots to emphasize the strong correlation. The noise in the 467- and $999-\mathrm{Hz}$ channels was at saturation prior to 0236 UT except for a brief interval and was initially anticorrelated with the density enhancements.

780- $\gamma$ magnetic field. For this field strength and an average ion density of $10 \mathrm{~cm}^{-3}$ in the enhancement, we obtain a wavelength of $160 \mathrm{~km}$ at a frequency of $100 \mathrm{~Hz}$ for a parallel propagating wave. While the wavelength would be somewhat less for off-angle propagation and somewhat less at the peak of the enhancement where the density is about $20 \mathrm{~cm}^{-3}$, we cannot escape the conclusion that the wavelength is comparable with the thickness of the enhancement and hence leakage is possible.

This conclusion is consistent with the observation that the enhancement at $0239 \mathrm{UT}$, which is about twice the dimension of the 0233 UT feature, contains some $100 \mathrm{~Hz}$ noise but much less than the 0237 UT enhancement which is three times as big again. On the other hand, the 0256 UT enhancement, which is even wider than the one at $0239 \mathrm{UT}$, contains no detectable ELF noise. Thus, while some of the behavior of the ELF signals can be explained in terms of the ability of the enhancements to duct the waves, not all the properties can.

The attenuation of the high latitude hiss in the density enhancements is not readily explained. While the lower hybrid resonance frequency lies well below the $467-\mathrm{Hz}$ channel, and thus the $467-$ and $999-\mathrm{Hz}$ waves can propagate only within a broad cone of directions about the direction of the magnetic field, this limitation does not seem to us sufficient to explain the observed attenuation.

Finally, we note that this close association between narrow band ELF noise at $100 \mathrm{~Hz}$ and plasma enhancements has been found on many orbits. In fact, on some orbits where the ELF data have been examined first, the plasma enhancements were located from the ELF data (K. W. Chan, personal communication, 1973).

4.2. Energetic electron observations. The possibility that the ELF signals are produced by 
Doppler-shifted electron cyclotron resonance can be further tested by reference to the flux of energetic (greater than $50 \mathrm{kev}$ ) electrons measured by the UCLA Energetic Electron Spectrometer. Before examining the flux of these energetic electrons, it is well to note that the presence of density enhancements beyond the plasmapause leads to resonance conditions which can be somewhat surprising. In Figures 8 and 9 , the minimum resonant energy at the dipole equator, labelled $L$, is plotted for both protons and electrons for different assumed plasma densities and for $\omega / \Omega=1 / 6$. Beyond the plasmapause, for example in the region $3.5<L<7.5$, $40 \mathrm{kev}$ electrons are stable to the electron resonance for a plasma density of $1.0 \mathrm{~cm}^{-3}$, but if the density rises to $10 \mathrm{~cm}^{-3}$, the same $40 \mathrm{kev}$ electrons can be unstable beyond $L=5$, while a density of $100 \mathrm{~cm}^{-3}$ is sufficient for these electrons to exceed the mini-

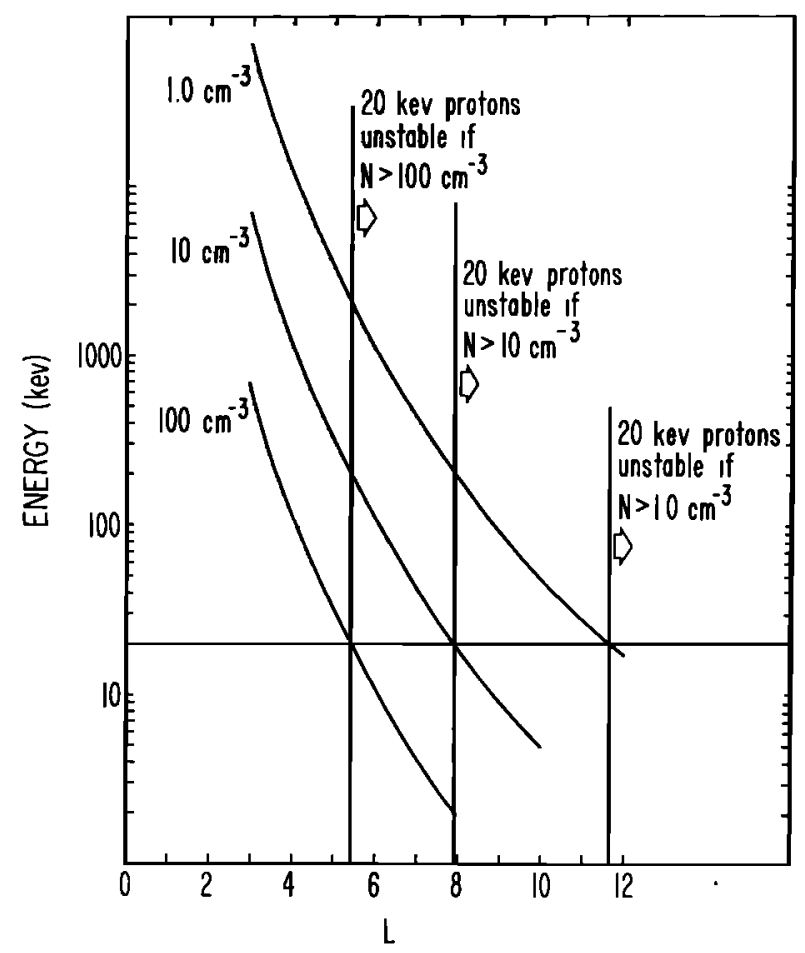

Fig. 8. Resonant energies for proton cyclotron instabilities at densities 1, 10, and $100 \mathrm{~cm}^{-3}$ at the dipole equator plotted against $L$. Wave growth can occur only if there exists a sufficiently large number of charged particles with energies above the resonant energy indicated by the applicable curve. The curves have been calculated for a wave frequency equal to $1 / 6$ of the equatorial proton gyrofrequency. This corresponds to a critical anisotropy of $\mathbf{0 . 2}$. For larger anisotropy the instability can occur at a lower energy.

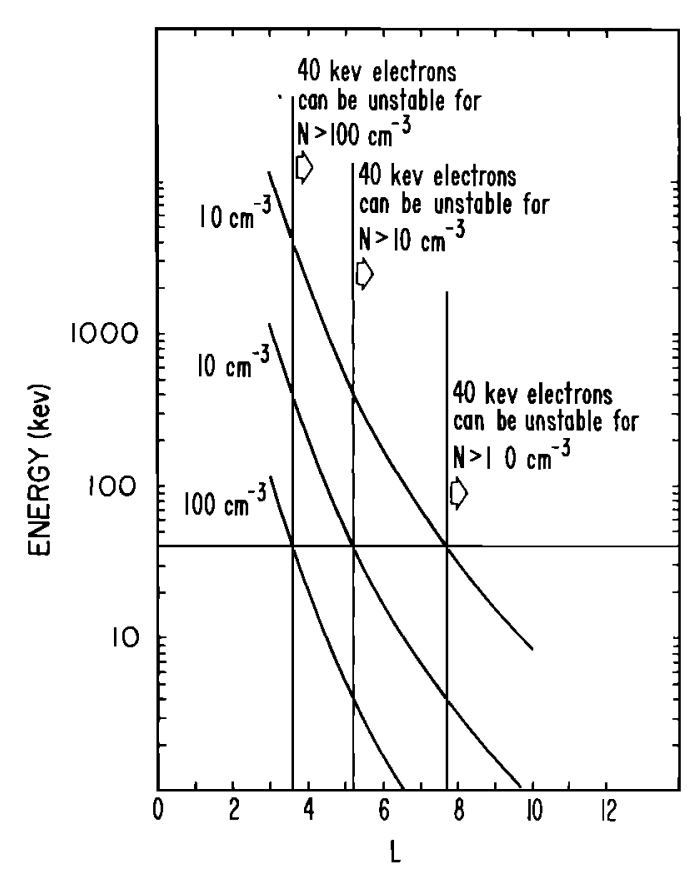

Fig. 9. Same as Figure 8 for electron cyclotron instabilities, calculated for a wave frequency equal to $1 / 6$ of the equatorial electron gyrofrequency.

mum resonance energy beyond $L=3.5$. Similar statements apply to ring current protons: $20 \mathrm{kev}$ protons at $L=6.6$ fail to meet the resonance criterion for densities of $10 \mathrm{~cm}^{-3}$ but can be resonant if the density is $100 \mathrm{~cm}^{-3}$.

On September 13,1968, plasma enhancements with densities from 10 to $30 \mathrm{~cm}^{-3}$ were encountered near $L=6$. From Figure 9 it is apparent that electrons of energy near $50 \mathrm{kev}$ lie just above the minimum energy required for resonance near the equator.

Figure 10 shows, for the same time interval, the integral directional flux of locally mirroring electrons of energy exceeding $50 \mathrm{kev}$ measured by the UCLA Energetic Electron Spectrometer. Resonant energies for the whistler-mode instability have been evaluated from the cold plasma theory:

$$
E_{\mathrm{li}}=\left(B^{2} / 8 \pi N\right)\left(\Omega_{e} / \omega\right)\left[1-\left(\omega / \Omega_{e}\right)\right]^{3}
$$

where $\Omega_{e}$ is the electron cyclotron frequency. The magnetic field, $B$, at the equator has been obtained from the locally measured magnetic field by assuming the ratio applicable for a dipole field line. The equatorial plasma density, $\mathrm{N}$, has been estimated assuming diffusive equilibrium of the plasma in a flux tube, an assumption which is appropriate if 
the plasma was detached from the plasmasphere, where it was in diffusive equilibrium, by the mechanism described previously. For such equilibrium, the dependence of density on magnetic latitude is weak, so the locally measured values were used for equatorial densities.

For the electron cyclotron mode to be unstable, the flux of electrons above the resonant energy must be sufficiently large that wave growth can exceed loss, a criterion which reduces to the requirement that the flux must exceed the maximum stably trapped flux, $J^{*}\left(>E_{R}\right)$. This flux has been estimated from an approximation of Kennel and Petshek [1966] as:

$$
\begin{aligned}
J^{*}\left(>E_{R}\right)=\left[\left(1-\omega / \Omega_{e}\right) /\left(A-\omega / \Omega_{e}\right)\right] \\
\cdot(3 B / 4 \pi L) 10^{10} \mathrm{~cm}^{-2} \sec ^{-1} \text { ster }^{-1}
\end{aligned}
$$

where $A$ is the anisotropy of the energetic electron distribution, positive for a distribution peaked at $90^{\circ}$. Although the values estimated for the anisotropy from the energetic particle measurements exceeded $1 / 4$, the value $A=1 / 6$ was used in the calculation to provide a conservative estimate, i.e., an overestimate, of the maximum stably trapped flux. The measured flux above the resonant energy exceeded the calculated stably trapped flux in each of the intervals during which $100-\mathrm{Hz}$ noise was present. The diagram shows the calculated $J^{*}\left(>E_{R}\right)$ and the observed flux above the resonant energy for several times during the interval. At $0233 \mathrm{UT}$, during the first isolated spike, the resonant energy was $108 \mathrm{kev}$, and the measured flux above that energy was too close to the estimated stably trapped flux to draw clear conclusions. In the density en-

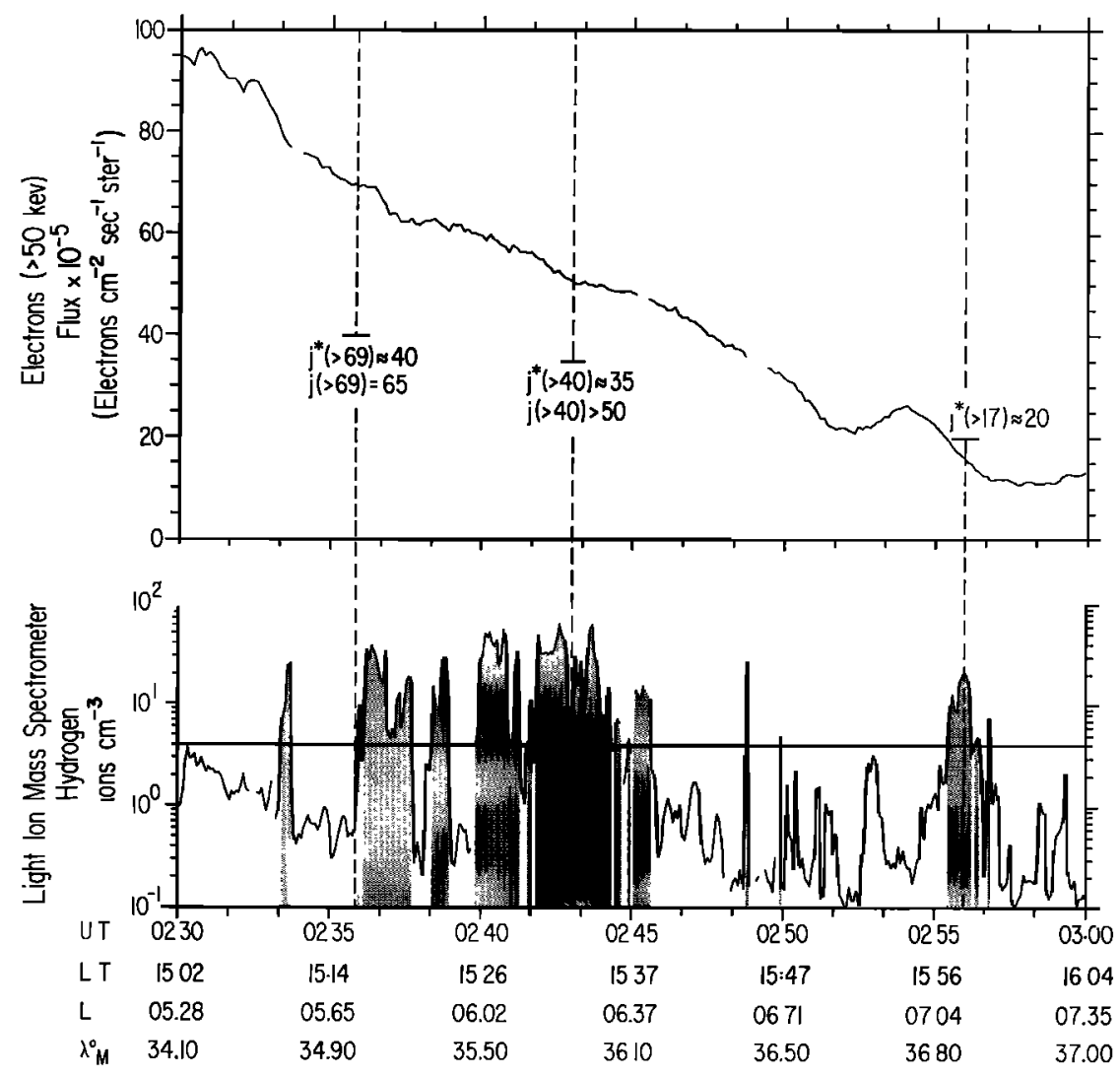

Fig. 10. Hydrogen ion density measured by the Lockheed Light Ion Mass Spectrometer and integral directional flux of $>50 \mathrm{kev}$ locally mirroring electrons measured by the UCLA energetic electron spectrometer on September 13, 1968. The minimum resonant energies have been determined for the density peaks just to the right of the vertical dashed lines at $0236.5,0243$, and $0256 \mathrm{UT}$, and estimates of the theoretical maximum stably trapped flux and the measured flux above these energies is shown. Although the observed energetic electrons satisfy the instability conditions, their distribution has not been visibly affected. 
hancement at $0256 \mathrm{UT}$, the resonant energy had dropped to $17 \mathrm{kev}$ and the available measurements were insufficient to provide an explanation for the absence of noise.

Note that although the energetic electrons whose flux is shown are unstable to whistler turbulence, they have not been significantly affected. No holes in their distribution develop in the plasma enhancements as a result of the precipitation of a large fraction of the resonant electrons. A comparison of time scales for spatial drift and for pitch-angle diffusion makes it clear that holes cannot develop. Plasma enhancements extend for 1 to $2 R_{E}$ at the equator. Gradient drift causes electrons of $50 \mathrm{kev}$ to traverse $1 R_{E}$ in less than $12 \mathrm{~min}$ for $\mathrm{L}$ between 5 and 10. Pitch-angle diffusion lifetimes can be calculated for the observed ELF power and are found to exceed one hr. Therefore, the plasma enhancement affects the electron flux very little. This argument is further supported by the observation that the energy density in the whistler waves is less than $1 \%$ of the particle energy density.

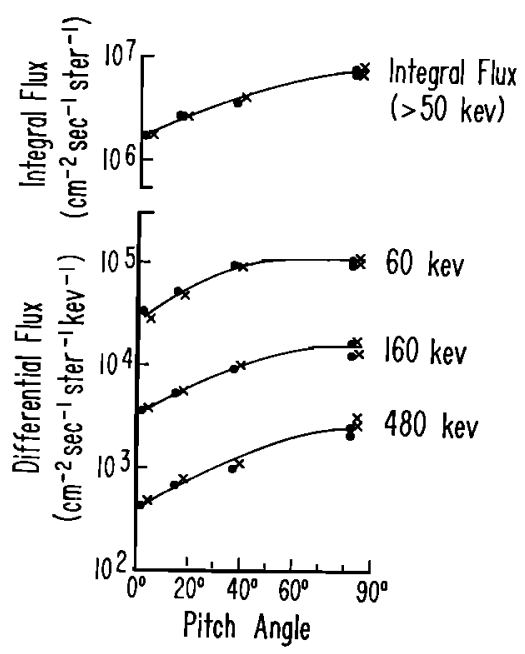

Fig. 11. Integral (top panel), and differential directional flux of energetetic electrons versus pitch angle, September 13, 1968. The measurements outside the plasma enhancement (0235.5 UT) are plotted as $x$, while those inside (0236.5 UT) are plotted as $\bullet$, and these points have been shifted $3^{\circ}$ towards smaller angles for greater clarity. Experimental uncertainty can be estimated from the spread in a pair of dots or crosses, which are measured by a pair of back-to-back detectors at the same pitch angle.
Further evidence that the onset of cyclotron resonance affects the energetic electrons very little is obtained from an examination of the pitch-angle distribution of the particles before and after the plasma enhancement was encountered at 0236 UT. Figure 11 shows pitch-angle distributions measured half a minute before and half a minute after the plasma enhancement was encountered. The top trace displays the integral flux of electrons of energy $>$ $50 \mathrm{kev}$ plotted versus pitch angle. Note that the flux peaked at $90^{\circ}$ as required for unstable wave growth. In the lower traces, the pitch-angle distribution of differential flux at selected energies is displayed. Flux measurements outside the plasma are marked $X$, and measurements inside the plasma are marked as dots, but have been shifted $3^{\circ}$ to the left to increase visibility. Experimental uncertainty is indicated by the separation of a pair of $X$ 's or of dots near $90^{\circ}$, and within this uncertainty there is no difference in the pitch-angle distributions or the energy spectra inside and outside of the density enhancements.

4.3. ELF electric fluctuations. The electron plasma frequency emission is sometimes detected in this region by the TRW Electric Field Experiment and in these cases confirms the plasma density reported by the Light Ion Mass Spectrometer. Low frequency electric fields are also measured, and at $200 \mathrm{~Hz}$ the electric field from this experiment correlated closely in intensity with the plasma density enhancements. The data of this experiment are shown in the central portion of Figure 12 with the plasma density shown below and the search coil amplitude above. The electric fields are measured every $9 \mathrm{sec}$ over a narrow range of frequencies $( \pm 20 \mathrm{~Hz})$ about $200 \mathrm{~Hz}$. The ratio of the electric to the magnetic field at $200 \mathrm{~Hz}$ was highly variable and the question of whether, in some regions, the fields are components of whistlers is being investigated. In other regions the electric field may represent purely electrostatic waves associated with the lower hybrid resonance frequency which increased and decreased, crossing through the pass band of the $200-\mathrm{Hz}$ channel as the density enhancements were traversed.

4.4. ULF magnetic fluctuations. For five orbits on which plasma enhancements were present outside the plasmapause, the magnetic field measured by the UCLA Fluxgate Magnetometer has been examined for evidence of ULF waves. The waves would have been identified if their peak-to-peak 


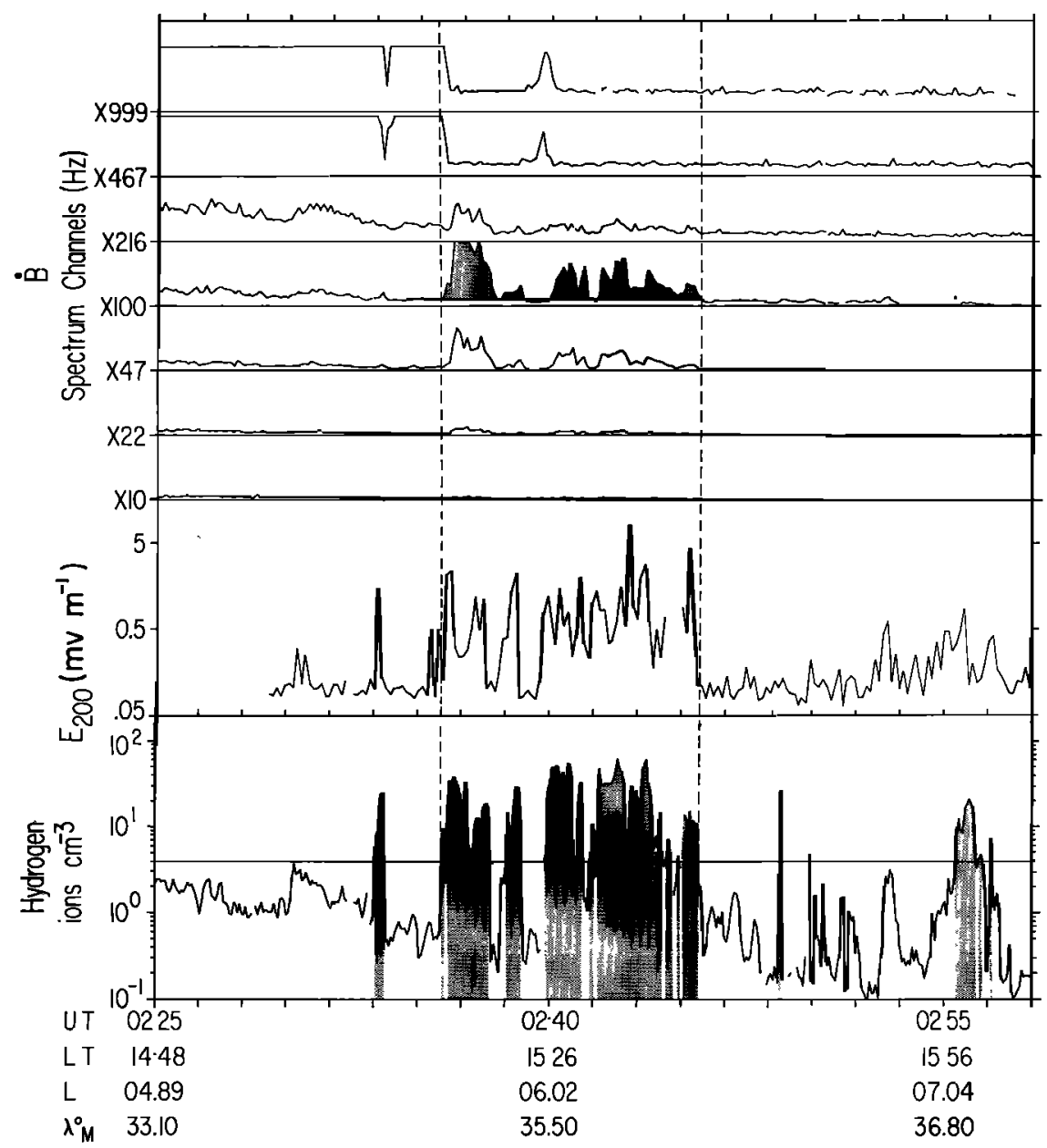

Fig. 12. Electric field amplitude of the $200-\mathrm{Hz}$ channel of the TRW Electric Field Experiment on OGO-5 is shown in the second panel from the bottom. Other panels repeat data of Figure 7 for comparison.

amplitude exceeded $2 \gamma$ and had periods of $0.5 \mathrm{sec}$ to $1 \mathrm{~min}$. A range of periods between 2 and $4 \mathrm{sec}$ is obscured by the presence of boom vibrations. ULF waves were observed on only two of the five orbits, and were present intermittently for a total of 12 min most frequently with a period of about $20 \mathrm{sec}$. Figure 13 shows examples of the ULF waves observed on the outbound pass of August 10, 1968, for which the thermal ion density is shown at the bottom. The density enhancement peaked between $L=7$ and $L=8.5$, near 1900 LT. At the top of the diagram on the right an example of the 20 -sec waves is shown. This wave was observed at $L=8.3$ and at 1913 LT. The diagram is $2 \mathrm{~min}$ across and the arrows are separated by $19 \mathrm{sec}$. The coordinate system is field-aligned, with the $z$ axis along the average magnetic field. The perturbations are purely transverse, and the wave is left-hand circularly polarized. For proton cyclotron resonance, the estimated resonant energy of a proton at the equator is about $50 \mathrm{kev}$, but no measurements are available to indicate what fluxes of protons of this energy were present.

On the top of the figure to the left, an example of an irregular burst of approximately 5-sec period at $L=7.7$ is shown. The diagram is $70 \mathrm{sec}$ across and the arrows are separated by $5 \mathrm{sec}$. The perturbation, again in the field-aligned coordinate system, is transverse but almost linearly polarized.

4.5. Summary. This introductory examination of phenomena observed in and near naturally occurring plasma enhancements raises many questions that should be examined further. For example, does cold plasma theory correctly predict the minimum 


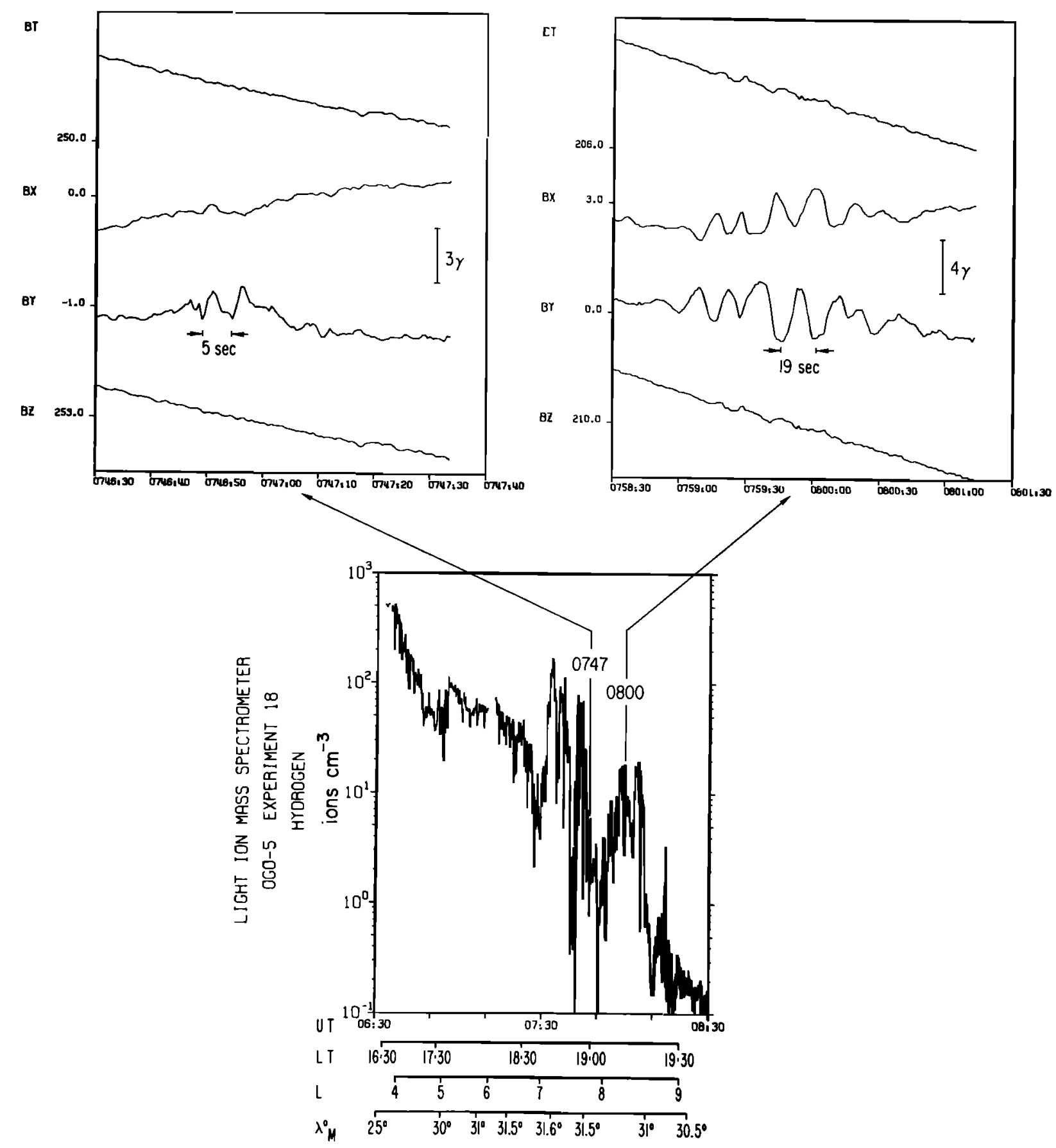

Fig. 13. Hydrogen ion density measured on OGO-5 on August 10, 1968 is plotted in the lower portion of this diagram. The upper panels show examples of the approximately 20-sec period waves (right-hand side) and the approximately 5-sec irregular bursts observed on this pass. Both upper panels are plotted in a field-aligned coordinate system in which the average field direction is $z$.

flux of energetic particles which must be exceeded for ELF wave growth? Does it correctly predict the required pitch-angle anisotropy? What is the critical size for ELF wave ducting? Why are ULF waves only occasionally present? What is the nature of the ELF electric noise observed? What are the conditions necessary for the formation of these detached plasma regions and what is their relationship to substorms? Is there an identifiable instability which produces striations within the cold plasma? 
We feel many of these questions can be answered by examining data we have already in our data libraries, and we hope to do so in future studies.

\section{CONCLUSION}

Active experiments will continue to contribute in important ways to our understanding of the magnetosphere. While the decade of the 1960's was dominated by passive experiments, the most significant contributions in the 1970 's may result from an increased emphasis on active experiments. Studies presently underway for utilization of the Space Shuttle indicate that the trend toward active experimentation may continue well into the 1980 's. The injection of light ion plasma into the outer radiation zone is one of the most promising of presently proposed experiments. However, many of the physical phenomena of interest in a plasma-seeding experiment can be studied in naturally occurring regions of enhanced plasma density. These regions should be probed extensively so that their properties are fully understood. The emphasis in planning artificial plasma-seeding experiments should then be placed on investigating those problems for which active experimentation is the unique available approach.

Acknowledgments. We are deeply indebted to C. R. Chappell, R. E. Holzer and F. L. Scarf who have freely provided us with their data and advice throughout the course of this study, and to P. J. Coleman, Jr., and T. A. Farley for their efforts in designing and building the OGO-5 experiments and their subsequent assistance in the analysis of the data. We are also grateful to W. $\mathrm{H}$. Campbell and H. I. West for providing us with data in advance of publication but which we did not have occasion to use in the events studied here. We also wish to thank R. K. Burton, and K. W. Chan for many stimulating discussions about the search coil data, and A. C. Fraser-Smith for pointing out that some enhancements may be too narrow to duct ELF signals effectively. This work was supported by the National Aeronautics and Space Administration under contracts NAS 5-9097 and NAS 5-9098 and grant NGR 05-007-305, and by the Office of Naval Research under contract N00014-73-C-0130.

\section{REFERENCES}

Barish, F. D., and J. G. Roederer (1972), Experimental test of magnetospheric models (abstract), EOS Trans. $A G U, 53,484$.

Brence, W. A., H. Neuss, R. E. Carr, and J. C. Gerlach (1972), An overview of the NASA/Max Planck Institute Project to study a barium release at five earth radii (abstract), EOS Trans. AGU, 53, 482.

Brice, N. (1970), Artificial enhancement of energetic particle precipitation through cold plasma injection: a tech- nique for seeding substorms?, J. Geophys. Res., 75, $4890-4892$.

Brice, N. (1971a), Space weather modification (abstract), EOS Trans. AGU, 52, 330.

Brice, N. (1971b), Harnessing the energy in the radiation belts, J. Geophys. Res., 76, 4698-4701.

Brice, N. M. (1972), Magnetospheres (abstract), EOS Trans. $A G U, 53,1106$.

Burton, R. K., R. E. Holzer, and E. J. Smith (1972), ELF hiss in the magnetosphere (abstract), EOS Trans. AGU, 53,490 .

Cartwright, D. G., and P. J. Kellogg (1970), Observations of plasma waves from the electron echo experiment (abstract), EOS Trans. AGU, 51, 805.

Chan. K. W., R. E. Holzer, and R. K. Burton (1972), ELF hiss associated with detached ion density enhancements (abstract), EOS Trans. AGU, 53, 1094.

Chappell, C. R. (1972), Recent satellite measurements of the morphology and dynamics of the plasmasphere, Rev. Geophys. Space Phys., 10, 951-979.

Chappell, C. R. (1973), Morphology of detached cold plasma regions in the magnetosphere, paper presented at Chapman Memorial Symposium on Magnetospheric Motions, American Geophysical Union, Boulder, Colo., June 1973.

Chappell, C. R., K. K. Harris, and G. W. Sharp (1971). The dayside of the plasmasphere, J. Geophys. Res., 76, 7632-7647

Cornwall, J. M. (1972), Precipitation of auroral and ringcurrent particles by artificial plasma injection, Rev. Geophys. Space Phys., 10, 993-1002.

Cornwall, J. M., and M. Schulz (1973), Theoretical aspects of artificial plasma injection, Aerospace Rep. ATR-73 (72 79)-1, 118 pp., The Aerospace Corporation, El Segundo, Calif.

Davis, T. N., W. N. Ness, M. C. Trichel, and E. M. Wescott (1973), Initial results of a recent electron accelerator experiment (abstract), EOS Trans. AGU, 54, 436.

Dowden, R. L., N. R. Thomson, L. E. S. Amon, D. A. McPherson, H. C. Koons, and M. H. Dazey (1973), Experimentally observed dependence of whistler propagation on pulse length (abstract), EOS Trans. AGU, 54, 423.

Foppl, H., G. Haerendel, J. Loidl, R. Lüst, F. Melzner, B. Meyer, H. Neuss, and E. Rieger (1965), Preliminary experiments for the study of the interplanetary medium by the release of metal vapour in the upper atmosphere, Planet. Space Sci., 13, 95-114.

Fraser-Smith, A. F. (1973), Generation of micropulsations with a large ground-based current loop (abstract), EOS Trans. $A G U, 54,419$.

Fu, J. H. M., H. M. Peek, and E. P. Marram (1973), Payload performance of the shaped-charge barium injection experiment OOSIK (abstract), EOS Trans. $A G U$, $54,432$.

Goldman, M. V., and D. F. DuBois (1971), Theory of parametric instability in the ionosphere (abstract), EOS Trans. $A G U, 52,883$.

Haerendel, G. (1971a), Observation of plasma convection in a westward traveling surge (abstract), EOS Trans. $A G U, 52,894$. 
Haerendel, G. (1971b), Interaction of barium clouds with the ionosphere (abstract), EOS Trans. AGU, 52, 881.

Haerendel, G., and R. Lüst (1970), Electric fields in the ionosphere and magnetosphere, in Particles and Fields in the Magnetosphere, edited by B. M. McCormac, pp. 213-228, Springer, New York.

Haerendel, G., R. Lüst, and E. Rieger (1967), Motion of artificial ion clouds in the upper atmosphere, Planet. Space Sci., 15, 1-18.

Haerendel, G., P. C. Hedgecock, and S.-I. Akasofu (1971), Evidence for magnetic field-aligned currents during the substorms of March 18, 1969, J. Geophys. Res., 76, 2382-2395.

Haerendel, G., H. Kappler, R. Lüst, E. Rieger, and H. Völk (1972), Momentum transfer from ambient magnetospheric to the seeded barium plasma (abstract), EOS Trans. $A G U, 53,483$.

Helliwell, R. A., and T. F. Bell (1971), Pulsation phenomena observed in long duration VLF whistler-mode signals, J. Geophys. Res., 76, 8414-8419.

Hendrickson, R. A., R. W. McEntire, and J. R. Winckler (1970), Electron echo data interpretation (abstract), EOS Trans. $A G U, 51,805$.

Hendrickson, R. A., J. R. Winckler, and R. L. Arnoldy (1973), Preliminary report on artificial magnetospheric electron injections at Fort Churchill (abstract), EOS Trans. $A G U, 54,436$.

Hess, W. N., M. C. Trichel, T. N. Davis, W. C. Beggs, G. E. Kraft, E. Stassinopoulos, and E. J. R. Maier (1971), Artificial auroral experiment: experiment and principal results, J. Geophys. Res., 76, 6067-6081.

Katsufrakis, J. P., C. G. Park, and D. L. Carpenter (1973), Detection of the plasmapause using VLF transmitter signals observed on polar orbiting satellites (abstract), EOS Trans. $A G U, 54,416$.

Kavanagh, L. D., Jr., S. W. Freeman, Jr., and A. J. Chen
(1968), Plasma flow in the magnetosphere, J. Geophys. Res., 73, 5511-5519.

Kennel, C. F., and H. E. Petschek (1966). Limit on stably trapped particle fluxes, J. Geophys. Res., 71, 1-28.

Kivelson, M. G., C. T. Russell, K. W. Chan, and C. R. Chappell (1972), An investigation of regions of high density cold plasma in the outer magnetosphere (abstract), EOS Trans. AGU, 53, 1103.

Mozer, F. S. (1972), Balloon measurements of ionospheric electric fields and precipitating particles during a barium cloud release in the magnetosphere (abstract), EOS Trans. $A G U, 53,483$.

Rieger, E., G. Haerendel, H. Kappler, J. Loidl, R. Lüst, and $H$. Neuss (1972), Magnetospheric convection derived from barium cloud motions at $5 R_{E}$ altitude (abstract ), EOS Trans. $A G U, 53,482$.

Schutz, S. R., F. S. Mozer, and G. Adams (1971), Electric field measurements in and near large barium releases in the ionosphere (abstract), EOS Trans. AGU, 52, 880.

Wescott, E. M., H. M. Peek, H. C. S. Nielsen, W. B. Murcray, R. J. Jensen, and T. N. Davis (1972), Two successful geomagnetic field-line tracing experiments, J. Geophys. Res., 77, 2982-2986.

Wescott, E. M., H. M. Peek, P. Bottoms, E. R. Rieger, H. C. Stenbaek-Nielsen, W. B. Murcracy, and T. N. Davis (1973), Results of 5 successful magnetospheric probings with barium shaped-charge generated plasma jets (abstract), EOS Trans. $A G U, 54,435$.

Winckler, J. R., R. A. Hendrickson, and R. W. McEntire (1970), Electron echo experiment-objectives of instrumental configuration (abstract), EOS Trans. $A G U, 51$, 805 .

Zhulin, I. A., V. I. Karpman, and R. Z. Sagdeev (1972), - Controlled experiments in the earth's magnetosphere, in Critical Problems of Magnetospheric Physics, edited by E. R. Dyer, pp. 245-260, IUCSTP Secretariat, National Academy of Sciences, Washington, DC. 